\title{
Die digitale Transformation als reflexiver turn: Einführende Literatur zur digitalen Geschichte im Überblick
}

\author{
Mareike König (iD
}

Angenommen: 24. September 2020 / Online publiziert: 24. November 2020

(C) Der/die Autor(en) 2020

Zusammenfassung Die digitale Transformation prägt seit einigen Jahrzehnten die Art und Weise, wie Geschichtswissenschaft betrieben, geschrieben, verbreitet, diskutiert und gelehrt wird. Dabei ist die Spannbreite dessen, was sich unter dem Sammelbegriff „Digitale Geschichte“ summieren lässt, riesig: Sie reicht von digitalen Praktiken, wie sie im Computerzeitalter für alle Forschenden alltäglich geworden sind, über Digitalität als Thema, bis hin zum Einsatz digitaler Methoden und Werkzeuge für die historische Erkenntnisgewinnung. Das an den Rändern offene transdisziplinare Praxisfeld ist zudem allein durch technische Entwicklungen im ständigen Fluss. Sehr unterschiedlich sind folglich Einführungen in die digitale Geschichte oder in die Digital Humanities, von denen in diesem Beitrag einige deutsch-, englisch- und französischsprachige Bände besprochen werden. Neben einer Einordnung dieses speziellen Literaturgenres zwischen Theorie und Praxis liegt der Fokus dabei zum einen auf den Änderungen von Forschung, Lehre, Kommunikation und Publikation durch die Digitalität. Zum anderen werden gegenwärtige Diskussionen und Kontroversen in und um die digitale Geschichte skizziert, wie sie zugleich in anderen Publikationen verhandelt werden.

Schlüsselwörter Digitale Geschichtswissenschaft · Digital turn · Digital Humanities

\footnotetext{
M. König ( $\varangle)$

Deutsche Geisteswissenschaftliche Institute im Ausland, Deutsches Historisches Institut Paris, Max Weber Stiftung, Paris, Frankreich

E-Mail: mkoenig@dhi-paris.fr
} 


\title{
The Digital Transformation as a Reflexive Turn: an Overview of Introductory Literature on Digital History
}

\begin{abstract}
The digital turn fundamentally questions the way in which knowledge is created, evaluated, taught, communicated and published. The range of what can be summed up as "digital history" is enormous: it extends from everyday digital practices, as they have become commonplace for all researchers, to the digital as a subject, to the use of digital methods and tools to explore data and make arguments. The paper provides a selective overview of some German, French and English introductions to digital history. In addition to thoughts on this particular literary genre between theory and practice, the focus is on the changes research has undergone as well as on current controversies around digital history.
\end{abstract}

Keywords Digital History $\cdot$ Digital turn $\cdot$ Digital Humanities

Die digitale Transformation der Welt hat die Geschichtswissenschaft nicht ausgespart und prägt seit einigen Jahrzehnten die Art und Weise, wie Forschung betrieben, geschrieben, verbreitet, diskutiert und gelehrt wird. Die Wurzeln reichen dabei zurück in die 1960er und 70er Jahre, als mithilfe von Lochkarten und Großcomputern umfangreiche statistische Daten in der Sozial- und Wirtschaftsgeschichte ausgewertet wurden, was zu heftigen Auseinandersetzungen im Fach führte. Ein weiterer Wurzelstrang der digitalen Geschichte kommt seit den 1980er Jahren aus der historischen Fachinformatik, die insbesondere in Deutschland prägend war. ${ }^{1}$ In den USA ist die digital history des entstehenden Internet der 1990er Jahre eng mit der public history verknüpft, zielten dortige Projekte doch vor allem auf die OnlinePräsentation von Quellen sowie auf nichtlineare Erzählweisen, auch für eine breite Öffentlichkeit. Die Publikation, Erschließung und Verknüpfung von Quellen über Digitalisierung, Volltexterkennung oder digitale Editionen sind weiterhin zentrale Handlungsbereiche der digitalen Geschichte. Während die meisten Alltagspraktiken von Historikerinnen und Historikern digital geworden und neue Formen der Publikation und Kommunikation entstanden sind, nutzt eine kleinere Anzahl an Spezialist_innen zudem computergestützte Methoden zur Exploration oder Interpretation von digitalisierten oder genuin digitalen Quellen, seien es Texte, Objekte, Abbildungen, Audio- oder Videoquellen, und trägt damit zu einer Vervielfachung der Wege der historischen Wissensgenerierung bei.

Schon angesichts dieser hier nur unvollständig angerissenen Vielfalt digitaler Praktiken wird deutlich, dass die Spannbreite dessen, was sich unter dem Sammelbegriff „Digitale Geschichte“ summieren lässt, riesig ist. Noch umfangreicher wird es, wenn Publikationen der Digital Humanities (DH) einbezogen werden, mit der die digitale Geschichte einige ihrer Methoden, Theorien und Gegenstände teilt. Die Ränder dieser Transdisziplin sind offen, das Feld trotz einer mittlerweile ansehnlichen Tradition allein schon durch technische Entwicklungen im ständigen Fluss.

\footnotetext{
1 Thaller, Manfred: From History to Applied Computer Science in the Humanities. Historical Social Research (HSR) Suppl. 29 (2017).
} 
Unterschiedliche Sprach- und Wissenschaftskulturen tragen darüber hinaus zu einer Pluralisierung der Digital Humanities bei, wie ein Blick auf die Website „Around DH in 80 Days “2 $^{\text {"2 }}$ und deren Neuauflage von 2020 mit einer Spezialisierung auf nicht-englischsprachige und minoritäre Gruppen weltweit zeigt. ${ }^{3}$ Je nachdem, ob der Schwerpunkt der Literatur auf praktischen Anleitungen, theoretischen Reflexionen, Beispielprojekten, den verschiedenen Methoden oder auf computergestützt erarbeiteten, thematischen Forschungsergebnissen liegt, hat man völlig unterschiedliche Bücher in den Händen, deren kleinster gemeinsamer Nenner hinter der Sammelbezeichnung DH zu verschwinden scheint. So anregend die Vielfalt ist, die als - häufig unbeachtetes - Gesprächsangebot innerhalb eines Fachs sowie zwischen den geisteswissenschaftlichen Disziplinen gesehen werden kann, so macht sie zugleich eine kohärente Gesamtübersicht oder eine thematisch verschränkte Sammelrezension zu Forschungsschwerpunkten und -lücken quasi unmöglich, selbst wenn der Fokus wie hier auf einführende Literatur und auf digitale Geschichtswissenschaft gelegt wird. Die Literatur veraltet zudem schnell, aktuelle Diskussionen finden eher in Journalaufsätzen oder in Wissenschaftsblogs statt, von wo aus einige Beiträge in überarbeiteter Form in klassische Publikationsformate gelangen. ${ }^{4}$ Aus diesen Gründen dürften monografische Einführungen selten und gemeinsam verfasste Handbücher oder Sammelbände eher die Norm sein.

Der folgende Rezensionsessay erhebt daher keinesfalls den Anspruch, das Feld in seiner Gänze vermessen zu wollen. Das Vorhaben ist sehr viel bescheidener und vermischt allgemeinere Reflexionen des Themas mit Kritik an Einzelbeiträgen, um darüber einen Überblick über die Entwicklung der digitalen Geschichte und einiger ihrer Kontroversen zu skizzieren. Nach einleitenden Beobachtungen zur Definition und zu fünf Gemeinsamkeiten der Literatur nimmt der Essay den Weg der konsekutiven Besprechung von zwei Lehrbüchern, zwei Einführungen in die digitale Geschichte und eines Praxis-Sammelbands zu den Digital Humanities, bevor in einem abschließenden Teil anhand von Aufsätzen aus weiteren DH-Sammelbänden Definitionsfragen erneut aufgegriffen und einige aktuelle Kontroversen in und um die digitale Geschichte skizziert werden. Da sich praktische Anleitungen zum Erlernen von IT-Kenntnissen sowie Vorstellungen digitaler Methoden kaum auf der Literaturebene beschreiben lassen, liegt der Schwerpunkt dieses Essays auf einer Gegenüberstellung der in den Publikationen vertretenen Annäherungen an die digitale Geschichte sowie auf den Änderungen, die darin für die Geschichtswissenschaft insgesamt gesehen werden.

\footnotetext{
2 Gil, Alex u. a.: Around DH in 80 Days, URL: <http://arounddh.elotroalex.com/> [Zugriff: 18.07.2020].

3 Global Outlook::Digital Humanities: Around DH 2020. Digital Humanities Projects in Languages Other than English or from Minoritized Groups Worldwide, URL: <http://arounddh.org/> [Zugriff: 18.07.2020].

4 In den Ausgaben der „Debates in the Digital Humanities“ von 2012 und 2016 haben Blogposts jeweils einen eigenen Abschnitt in den verschiedenen Themenkapiteln. Aber auch die anderen besprochenen Bände zitieren ausgiebig aus der Blogosphäre.
} 


\section{Offene Definitionen bei fünf Gemeinsamkeiten}

Gleich vorweg: eine einheitliche und generell akzeptierte Definition, was Digital Humanities oder digitale Geschichte eigentlich genau sind und worin sie sich unterscheiden, existiert nach wie vor nicht. Mit der enormen Breite und Fluidität dieses transdisziplinären Praxisfelds wurden die wesentlichen Gründe dafür bereits genannt. Auch bei den hier besprochenen Werken sind die Definitionsangebote unterschiedlich. Neu ist jedoch, dass bei keinem Band Definitionsfragen im Vordergrund stehen, worauf im Ausblick kurz eingegangen wird. Das zeugt von einem größeren Selbstbewusstsein der DH, wobei die Definitionsschwierigkeiten, die es in anderen Disziplinen genauso gibt, auch positiv und als begrüßenswerte Reflexivität der Methoden und Gegenstände des Feldes gesehen werden können. ${ }^{5}$ Zugleich spiegelt sich darin die Tatsache, dass manche Kontroverse um die DH zwar nicht auf dem Papier, wohl aber in der Praxis durch die Einrichtung von Lehrstühlen, Studiengängen, Fachzeitschriften, Tagungen, Verbänden, Förderprogrammen et cetera entschieden ist. ${ }^{6}$ Darüber hinaus kann die Offenheit in der Definition auch als Strategie gedeutet werden, die Grenzen des digitalen Möglichkeitsraums bewusst flexibel zu halten, um damit Anschlussfähigkeit zu signalisieren. ${ }^{7}$

Daran lassen sich trotz der eingangs erwähnten Unterschiede fünf generelle Beobachtungen anschließen, die gegenwärtig - oder auch schon etwas länger - den Grundtenor der Literatur über digitale Geschichte bilden: Dazu gehört erstens das durchgängig vorhandene Eingangsstatement - ob auf Deutsch, Englisch oder Französisch -, dass mittlerweile alle Historiker_innen digital Forschende sind, egal ob sie sich dessen bewusst sind und ob sie sich selbst als solche verstehen. ${ }^{8}$ Denn alle verwenden einen Computer für den Mailverkehr und die Textverarbeitung, sie betreiben Online-Recherche in Suchmaschinen, Bibliothekskatalogen oder in digitalen Sammlungen. Sie alle verwenden Online-Plattformen wie JSTOR für den Zugriff auf Journalartikel, fertigen in Archiven Fotos oder Scans von Quellen an, greifen online auf Rezensionen zur Fachliteratur über „H-Soz-Kult“, ,,sehepunkte“, ,FranciaRecensio“ und andere zu. Das Suchen und Arbeiten im ,unendlichen Archiv“ (infinite archive $)^{9}$ der riesigen Online-Sammlungen an digitalisierten Quellen fühlt sich mittlerweile ,so revolutionär [an] wie Haferbrei“ (as revolutionary as oatmeal $)^{10}$,

\footnotetext{
5 Plantin, Jean-Christophe: Les digital humanities. Accomplissements et défis pour un agencement postdisciplinaire, in: Les cahiers du numérique 10 (2014), H. 4, S. 41-62, hier S. 47, URL: <https://www.cairn. info/revue-les-cahiers-du-numerique-2014-4-page-41.htm> [Zugriff: 18.07.2020].

${ }^{6}$ So das Argument von Sahle, Patrick: Digital Humanities? Gibt's doch gar nicht!, in: Zeitschrift für digitale Geisteswissenschaften, Sonderband 1: Grenzen und Möglichkeiten der Digital Humanities, hrsg. v. Constanze Baum/Thomas Stäcker (2015), DOI: <https://doi.org/10.17175/sb001_004> [Zugriff: 18.07.2020].

7 Plantin: Digital humanities (wie Anm. 5), S. 47.

${ }^{8}$ So bereits 2006 von Cohen, Daniel J./Rosenzweig, Roy: Digital History. A Guide to Gathering, Preserving, and Presenting the Past on the Web, Pennsylvania UP, Philadelphia, PA 2005, URL: <http://chnm. gmu.edu/digitalhistory/> [Zugriff: 18.07.2020].

9 Turkel, William J./Kee, Kevin/Roberts, Spencer: A Method for Navigating the Infinite Archive, in: Weller, Tony (Hrsg.): History in the Digital Age, Routledge, London/New York 2013, S. 61-75.

${ }^{10}$ Putnam, Lara: The Transnational and the Text-Searchable. Digitized Sources and the Shadows They Cast, in: The American Historical Review 121 (2016), H. 2, S. 377-402, hier S. 380.
} 
trotz zahlreicher Zugangsbeschränkungen und einem Schwerpunkt auf textbasierten Quellen der Nationalgeschichte aus dem Globalen Norden. In jüngster Zeit zeichnen sich Versuche ab, größere begriffliche Klarheit in der digitalen Geschichte zu schaffen, indem das „digitalisierte“ Arbeiten - also die Suche und das Online-Lesen von Digitalisaten bei klassischer Interpretationsarbeit - unterschieden wird von Praktiken, bei denen mit computergestützten Methoden Analysen und Visualisierungen von Texten, Daten und Objekten durchgeführt wird (computational history). ${ }^{11}$

Zweitens zeichnet sich die Literatur über digitale Geschichte traditionell dadurch aus, dass sie das Neue und die Wichtigkeit digitaler Ansätze für die historische Forschung betont und dabei nicht immer die Gefahr vermeidet, in euphorische oder ahistorische Diskurse über ein vermeintliches Vorher/Nachher zu geraten. In jüngster Zeit verschiebt sich der Fokus dabei auf die Dringlichkeit, zumindest über Grundkenntnisse in digital literacy zu verfügen, um das digitalisierte Arbeiten methodisch sauber durchführen zu können. Denn diese Praktiken haben genau wie die Selektions-, Präsentations- und Indexierungsentscheidungen bei der Bereitstellung von Quellen Auswirkungen auf die Forschungsergebnisse, etwa durch Lücken im OCR eines Textes, durch fehlende Suchstrategien oder den Kontext-Kollaps bei der Arbeit in großen Quellensammlungen. ${ }^{12}$ Im Vergleich zu den 2000er und frühen 2010er Jahren sind dennoch, so meine subjektive Wahrnehmung, die kämpferischen und revolutionären Töne in der aktuellen digitalen Geschichtswissenschaft weniger ausgeprägt.

Daran anknüpfend gibt es drittens in der gegenwärtigen Literatur zur digitalen Geschichte die übereinstimmende Einschätzung - und das schon seit $2003^{13}$ -, dass neben digital literacy auch die Wichtigkeit der Beherrschung computergestützter Methoden zunehmen wird, allein angesichts der schieren Masse, Diversität und Fragilität an born-digital-Quellen der gegenwärtig erzeugten historischen Überlieferung. ${ }^{14}$ Aufgrund der großen Bandbreite an proprietären, freien und personenbezogenen Daten werden Kenntnisse der rechtlichen und ethischen Rahmenbedingungen als historisches Handwerkszeug mithin ebenso unerlässlich wie eine digitale Quellenkritik, die informationstechnische Prozesse berücksichtigt, um Authentizität und Integrität von Quellen verlässlich prüfen zu können. ${ }^{15}$ Nach wie vor treibt daher die meisten der hier besprochenen Arbeiten die Frage an, wie sie traditionell arbeitende Forschende von Sinn und Bedeutung digitaler Kenntnisse und

\footnotetext{
${ }^{11}$ Vgl. ebd., S. 379 sowie Laite, Julia: The Emmet's Inch. 'Small' History in a Digital Age, in: Journal of Social History 53 (2019), H. 4, S. 963-989.

12 Vgl. dazu Hitchcock, Tim: Academic History Writing and its Disconnects, in: Journal of Digital Humanities 1 (2011), H. 1, URL: <http://journalofdigitalhumanities.org/1-1/academic-history-writing-and-itsdisconnects-by-tim-hitchcock/> [Zugriff: 18.07.2020].

13 Rosenzweig, Roy: Scarcity or Abundance. Preserving the Past in a Digital Age, in: American Historical Review 108 (2003), H. 3, S. 735-762, PDF: <http://chnm.gmu.edu/digitalhistory/links/pdf/introduction/0. 6b.pdf> [Zugriff: 18.07.2020].

${ }^{14}$ Milligan, Ian: History in the Age of Abundance. How the Web is Transforming Historical Research, McGill-Queen's UP, Montreal 2019.

${ }^{15}$ Föhr, Pascal: Historische Quellenkritik im Digitalen Zeitalter, Werner Hülsbusch, Glückstadt 2019. Zugl.: Basel, Univ., Diss., 2017. URL der Online-Ausgabe der Dissertation: <https://edoc.unibas.ch/ 64111/> [Zugriff: 18.07.2020].
} 
Praktiken überzeugen können. Die Nützlichkeit von regulären Ausdrücken (regular expressions) etwa, die als Filterkriterien in der Textsuche verwendet werden können, wird nicht unmittelbar einleuchten, solange man ein klassisches Quellenstudium betreibt, sondern erst dann, wenn große Datenmengen durchsucht und bereinigt werden müssen. ${ }^{16}$ Somit richten sich die Texte häufig an ein doppeltes Publikum, an Expert_innen und traditionell arbeitende Forschende zugleich, ein durchaus geduldiger Vermittlungsversuch, der aber seit Jahrzehnten weitgehend ungehört verhallt, zum Schaden des Fachs und zum Nachteil des Nachwuchses. ${ }^{17}$

Dazu gehört viertens die ebenso durchgehend in der Literatur zu findende Einschätzung, dass es in der digitalen Geschichte keinen tatsächlichen Widerstreit zwischen qualitativen und quantitativen Ansätzen gibt. Es herrscht Übereinstimmung, dass die menschliche Interpretation die wichtigste Methode der Geschichtswissenschaft bleiben wird. Digitale Methoden sollen die alten nicht ersetzen, sondern diese ergänzen. Wichtig ist, dass in jedem Fall in einer Mischung aus digitalen und analogen Praktiken gearbeitet wird. ${ }^{18}$ Auch diese Einschätzung mag zur Beruhigung der nicht-digitalen Kolleg_innen und der Überwindung der Gräben aus der Zeit der cliometrics dienen. Ebenso übereinstimmend wird die Meinung vertreten, dass Algorithmen, Suchmaschinen, digitale Werkzeuge und Portale und deren Nutzung gleichwohl einen Einfluss auf Forschungsergebnisse haben. Dies bleibt freilich mangels Studien vage, und ob und wie tief damit epistemologische Änderungen des Fachs verbunden sind, wird verschieden thematisiert. Einig ist man sich wiederum - wie oben erwähnt - darin, dass das digitale Vorgehen transparent gemacht und methodisch reflektiert erfolgen muss im Sinne einer digital literacy. Als „digitale Hermeneutik“ oder ,geschichtswissenschaftliche Datenkritik“ bezeichnet dies Andreas Fickers, der damit den gesamten „Arbeits- bzw. Forschungsprozess einbezieht, der sich von der Fragestellung über die Informationssuche, Analyse, Interpretation bis zur Repräsentation und Darstellung neuen historischen Wissens erstreckt": digital tool criticism, data criticism, algorithmic criticism und interface criticism gehören folglich seiner Meinung nach als neue Kenntnisse in das Portfolio von Historiker_innen. ${ }^{19}$

Und als fünfte und letzte gemeinsame Beobachtung: Digital affine Forschende sind Verfechter von Open Access, lange bevor der freie Zugang auf publizierte Forschungsergebnisse politisch gewollt und weit verbreitet wurde. Dies spiegelt sich im Produktionsverfahren und im Publikationsmodus der meisten hier besprochenen

\footnotetext{
16 Crymble, Adam: Review of “Exploring Big Historical Data. The Historian's Macroscope”, in: Reviews in History, Februar 2016, URL: <https://reviews.history.ac.uk/review/1889> [Zugriff: 18.07.2020].

17 Hier erlaube ich mir, auf meinen eigenen Beitrag zu verweisen: König, Mareike: Geschichte digital - zehn Herausforderungen, in: Arendes, Cord u. a. (Hrsg.): Geschichtswissenschaft im 21. Jahrhundert. Interventionen zu aktuellen Debatten, De Gruyter, Berlin u. a. 2020, S. 67-76.

18 Zaagsma, Gerben: On Digital History, in: BMGN - Low Countries Historical Review 128 (2013), H. 4, S. 3-29, URL: <http://www.bmgn-lchr.nl/index.php/bmgn/article/view/9344> [Zugriff: 18.07.2020].

19 Fickers, Andreas: Update für die Hermeneutik. Geschichtswissenschaft auf dem Weg zur digitalen Forensik?, in: Zeithistorische Forschungen 17 (2020), H. 1, S. 157-168, URL: <https://zeithistorischeforschungen.de/1-2020/5823> [Zugriff: 18.07.2020]. Siehe auch van Zundert, Joris J.: Screwmeneutics and Hermenumericals. The Computationality of Hermeneutics, in: Schreibman, Susan/Siemens, Ray/ Unsworth, John (Hrsg.): A New Companion to Digital Humanities (Blackwell Companions to Literature and Culture), Wiley-Blackwell, West Sussex u.a. ${ }^{2} 2016$, S. 331-347.
} 
Bände, die häufig über ein Open-Peer-Review und damit in einem offenen Diskussionsprozess entstanden sind und neben dem gedruckten Buch zugleich als OpenAccess-Fassung angeboten werden. Zugleich setzen sie sich für eine offene Wissenschaftskommunikation in verschiedenen Formaten ein und für die Zugänglichmachung und Nachnutzung von Forschungsdaten, Code und Werkzeugen. Allerdings sind die beiden deutschsprachigen hier besprochenen Bände nun gerade nicht im Open Access publiziert. Daraus lässt sich natürlich kein Trend ableiten, aber es fällt zumindest auf.

\section{Zum Einstieg: zwei Lehrbücher}

Mit dem Buch „Exploring Big Historical Data. The Historian's Macroscope“ von Shawn Graham, Ian Milligan und Scott Weingart liegt seit 2016 ein erstes monografisches Lehrbuch vor, das sowohl Theorie als auch Praxis der digitalen Geschichte bedient. ${ }^{20}$ Das Buch ist im offenen Schreibprozess als Open-Peer-Review-Projekt entstanden. Eine erste Version des Textes wurde online veröffentlicht und zur Diskussion gestellt. ${ }^{21}$ Das gedruckte Buch stellt die überarbeitete Fassung dar. Graham und Milligan sind kanadische, Weingart ein US-amerikanischer Historiker; alle drei kamen schon früh mit Computern in Berührung und gehörten zu den Internetnutzern der ersten Stunde. Das rund 280 Seiten umfassende und sehr empfehlenswerte Buch ist in zwei einleitende und fünf praxisorientierte Kapitel gegliedert. Unter dem titelgebenden „Macroscope“ der Geschichtswissenschaft verstehen die Autoren sowohl ein Werkzeug als auch eine Perspektive auf Geschichte, bei der durch Reduzierung von Komplexität in großen Datenmengen verdeckte Muster und Verbindungen deutlich werden (S. 1). Den datengetriebenen Zugang auf die Geschichte sehen die Autoren nicht als einzig seligmachende Art und Weise für historische Erkenntnisgewinnung, sondern als festen Teil des zukünftigen „fachlichen Mosaiks“. Somit bekommen Computerkenntnisse für die Geschichtsforschung eine ähnliche Bedeutung wie Sprachkenntnisse: in einigen Fällen „,nice to have“, in anderen Fällen eine Selbstverständlichkeit und schlicht notwendig (S. 4). Digitale Geschichte impliziert aus Sicht der Autoren zugleich einen offenen und öffentlichen Zugang auf Forschungsdaten und -ergebnisse, um diese nachvollziehbar zu machen.

Einleitend widmen sich Graham, Milligan und Weingart der Frage, was Big Data für die Geschichtswissenschaft bedeutet. An mehreren Beispielen zeigen sie, wie Historiker_innen durch den Einsatz von computergestützten Methoden Erkenntnisse gewinnen und damit die Forschungsdiskussion bereichern konnten. Es folgen eine kurze Darstellung der Entwicklung der digitalen Geschichte und der Digital Humanities und deren Vorläufer Humanities Computing. Aus Sicht der Autoren prägt die Verbindung von quantitativer und computergestützter Geschichte der cliometrics der 1960er und 1970er Jahre bedauerlicherweise bis heute hartnäckig das falsche Bild

\footnotetext{
${ }^{20}$ Graham, Shawn/Milligan, Ian/Weingart, Scott: Exploring Big Historical Data. The Historian's Macroscope, ICP, London 2016.

${ }^{21}$ Dies.: Exploring Big Historical Data. The Historian's Macroscope, URL: <http://www.themacroscope. org/2.0/> [Zugriff: 18.07.2020].
} 
der digitalen Geschichte als eine Objektivität und Wahrheit suchende, positivistische Strömung (S. 23). Die Umbenennung von Humanities Computing in Digital Humanities 2004 durch den von Susan Schreibman, Ray Siemens und John Unsworth herausgegebenen Sammelband ${ }^{22}$ sei mit einer Ausweitung des Feldes einhergegangen, das seitdem unter anderem born-digital-Daten, Designfragen und Media Studies einschloss und den Schwerpunkt stärker auf Werkzeuge und weniger auf methodische Diskussionen legte. Auch sei damit die Betonung von „,computing“ auf die „humanities“ verlagert worden, also auf die Geisteswissenschaften und deren spezifische Epistemologie und Fragestellungen. Mit ihrer eigenen Definition der Digital Humanities bleiben Graham, Milligan und Weingart jedoch vage: Es gehe in Teilen darum, zu verstehen, was digitale Werkzeuge anzubieten haben, sowie vor allem darum, zu begreifen, wie sich das Digitale auf das Verständnis der Vergangenheit ausgewirkt habe und weiterhin auswirkt (S. 26). Trotz der Kürze enthält die Definition einen Schreibfehler mit der Doppelung eines Halbsatzes; vermutlich waren die Sätze mehrfach umgestellt worden, was symptomatisch für die häufig tastende Herangehensweise in Definitionsfragen der digitalen Geschichte ist.

Wie auch die meisten anderen hier besprochenen Autoren unterstreichen Graham, Milligan und Weingart, dass die Anwendung von computergestützten Methoden keine epistemologischen Änderungen für das Fach mit sich bringt. Nach wie vor arbeiten Historiker_innen mit Spuren der Vergangenheit. Die menschliche Interpretation bleibe als Herzstück der historischen Forschung erhalten, als subjektive Tätigkeit und als Praxis des narrativen Schreibens und Kreierens (S. 32 f.). Eine digitale Herangehensweise zwinge historisch Forschende aber dazu, Quellen und Materialien anders als bisher anzusehen und sich ihnen weniger suchend nach einem Beweis für eine These als stärker „,entdeckend“ zu nähern (S. 34).

Im zweiten ebenfalls noch überwiegend theoretischen Kapitel werden Schlüsselbegriffe der Digital Humanities definiert, darunter Open Access, Copyright, Data-Mining und Bezeichnungen aus der digitalen Textanalyse wie Worthäufigkeit, ngrams, keyword in context und so weiter. Ein längerer Abschnitt widmet sich der Frage, warum alle Historiker_innen digital Forschende sind (S. 46-50). Als Problem benennen Graham, Milligan und Weingart, dass historisch Forschende zwar digitale Werkzeuge in ihre überwiegend traditionelle Arbeitsweise integriert haben, dies aber zumeist unkritisch (S. 47). Genauso wenig werden der Einfluss von Algorithmen in Suchmaschinen oder die Auswirkungen von Fehlern und Lücken im Volltext einer digitalisierten Quelle auf die Forschungsergebnisse hinterfragt (S. 49), eine Gefahr, auf die vor allem Tim Hitchcock in seinen Publikationen immer wieder verweist. ${ }^{23}$ Damit wird aus meiner Sicht jedoch fraglich, ob sich die zuvor geäußerte Behauptung, es gäbe durch eine digitale Herangehensweise keine epistemologischen Änderungen in der Geschichtswissenschaft, in dieser Form aufrechterhalten lässt.

In den nachfolgenden Kapiteln beginnt der praktische hands-on-Teil des Buches, zunächst mit Tools zum web scraping und Data-Mining, um auf Webseiten publizierte Texte, Daten oder Dateien in einem Schwung herunterzuladen, anstatt sie zeit-

\footnotetext{
22 Schreibman, Susan/Siemens, Ray/Unsworth, John (Hrsg.): A Companion to Digital Humanities, Blackwell, Oxford 2004, URL: <http://www.digitalhumanities.org//companion/> [Zugriff: 18.07.2020].

23 Vgl. Hitchcock: Writing (wie Anm. 12).
} 
aufwendig einzeln per Rechtsklick zu speichern. Dabei steigt der Schwierigkeitsgrad langsam von webbasierten Anwendungen über reguläre Ausdrücke zum Säubern von großen Datensets bis zu den ersten eigenen Scriptzeilen in der Programmiersprache Python. Ein Kapitel ist topic modeling gewidmet als einer computergestützten Methode für die Inhaltsanalyse großer Textkorpora, die im Vergleich zur Volltextsuche vertiefte inhaltliche Einblicke ermöglicht. Dazu werden Themen (topics) in einem Korpus über Häufigkeiten oder Verteilung von Wörtern ermittelt und Wortgruppen angezeigt, die statistisch auffällig häufig miteinander vorkommen. Diese Wortgruppen sind nicht nur Indikatoren für mögliche Inhalte eines Korpus und deren Entwicklung, sie lassen sich auch mit anderen Korpora vergleichen. Um grundlegende Konzepte und Begriffe der Visualisierung geht es im fünften Kapitel, danach folgen zwei Kapitel zu Netzwerken als Mittel der Datenanalyse. Im abschließenden Kapitel verweisen Graham, Milligan und Weingart auf digitale Möglichkeiten, die eigenen Forschungsresultate zu verbreiten. Damit liegt der Schwerpunkt des Bandes auf der computergestützten Analyse textlicher Quellen. Historische Geografische Informationssysteme (GIS), die andernorts als Hauptbetätigungsfeld der digitalen Geschichte gelten $^{24}$, bleiben dagegen außen vor.

Das Lehrbuch ist in seiner Art bisher einmalig und zeichnet sich durch einen gelungenen Praxisteil aus. Dessen Stärke ist der anwendungsbezogene Aufbau. Es werden nicht einfach bezugslos Techniken erklärt, sondern im Mittelpunkt steht stets ein konkretes Problem, das gelöst, oder eine Fragestellung, die beantwortet werden soll. Viele der Beispiele sind der Online-Einführung „The Programming Historian“25 entnommen, die mittlerweile 82 technische Tutorials zum Programmieren unter anderem für die Bereiche web scraping, Datenanalyse, Netzwerkforschung, Mapping und digitales Publizieren enthält. Wer programmieren lernen möchte, kann das Buch und die Website parallel verwenden, auch wenn Versionsunterschiede bei Python die eine oder andere Übung erschweren. Die Lernkurve ist steil, der Zeitaufwand allerdings enorm, da man nicht einfach Buchseiten oder Teile einer Übung überspringen kann, wenn man etwas nicht verstanden hat oder etwas nicht klappt. Ein Nachteil solcher Lehrbücher ist ihre in technischer Hinsicht kurze Haltbarkeitszeit. Abhilfe schaffen könnte die vollständige Verlagerung des praktischen Teils ins Web. Damit ließe sich im vorliegenden Fall zugleich das Problem der zahlreichen Abbildungen und Screenshots lösen, die im gedruckten Buch nur in Schwarz-Weiß und bisweilen in schlechter Auflösung publiziert wurden, für das Verständnis der Übungen aber wichtig sind, zumal im Text die Farbgebung mit erläutert wird. ${ }^{26}$

Als weitere und dazu komplementäre Einführung können Interessierte seit 2017 ebenso auf die für sie passenden Kapitel im ersten deutschsprachigen Lehrbuch zu den Digital Humanities zurückgreifen, das Fotis Jannidis (Professor für Computerphilologie an der Universität Würzburg), Hubertus Kohle (Professor für Kunst-

\footnotetext{
${ }^{24}$ Vgl. Robertson, Stephen: The Differences Between Digital Humanities and Digital History, in: Gold, Matthew K./Klein, Lauren F. (Hrsg.): Debates in the Digital Humanities 2016, Minnesota UP, Minneapolis, MN/London 22016, S. 289-307, URL: <http://dhdebates.gc.cuny.edu/debates/text/76> [Zugriff: 18.07.2020].

25 The Programming Historian, URL: <https://programminghistorian.org/> [Zugriff: 18.07.2020].

26 Vgl. dazu die Rezension: Crymble: Review (wie Anm. 16).
} 
geschichte an der Ludwig-Maximilians-Universität München) und Malte Rehbein (Professor für Digital Humanities an der Universität Passau) gemeinsam herausgegeben haben. ${ }^{27}$ Vertreter_innen unterschiedlicher geisteswissenschaftlicher Fächer haben die präzisen Kurzeinführungen als für sich allein stehende Texte verfasst. Der Band ist als Einheit angelegt, was schon der Blick ins Inhaltsverzeichnis zeigt: Die Namen der Autorinnen und Autoren sucht man dort vergebens, vielmehr stehen die Inhalte und mithin die vorgeschlagene Gliederung der Digital Humanities als Feld im Mittelpunkt. Das Buch ist gleichermaßen nützlich für Studierende und Forschende, die sich einen Überblick über die vielfältigen Methoden und Anwendungen der DH verschaffen möchten. Dank eines Registers kann es zugleich als Nachschlagewerk dienen. ${ }^{28}$ Die Einführung ist in fünf Hauptkapitel unterteilt, die von „Grundlagen“ (darunter eine Geschichte der Digital Humanities), „Datenmodellierung“, „Digitale Objekte“ und „Digitale Methoden“ zu „Recht und Ethik“ reichen. Die Lektüre der Beiträge ist durchaus anspruchsvoll und setzt Vorwissen oder genügend Interesse voraus, das Gelesene zu vertiefen, etwa bei den gelungenen Kapiteln zum Aufbau des Computers von Harald Klinke ${ }^{29}$, zur Theorie digitaler Medien von Ramón Reichert ${ }^{30}$, zur Grundlage der Datenmodellierung von Fotis Jannidis ${ }^{31}$ oder zu Geografischen Informationssystemen von Armin Volkmann ${ }^{32}$. Mit den Aufsätzen im dritten Hauptkapitel unter anderem zu Digitalen Editionen, Digitalisierung, Bibliotheken, Archiven, Museen sowie zum Aufbau von Datensammlungen sind rund 80 Seiten den für die historische Forschung so wichtigen digitalen Objekten und ihrer Online-Bereitstellung gewidmet. Ebenso finden das digitale Publizieren und die digitale Wissensproduktion inklusive Crowdsourcing und kollaboratives Schreiben in Wikipedia in diesem Abschnitt Berücksichtigung. Zahlreiche Anwendungsbeispiele sowie praktische Anleitungen begleiten die theoretischen Überlegungen im Band. Auf der Website des Verlags wird zusätzlich ein elfseitiges PDF mit Übungsaufgaben publiziert ${ }^{33}$, was für einen Band der Digital Humanities allerdings etwas antiquiert anmutet.

Da die Artikel als selbstständige Einheiten angelegt sind, kommt es hin und wieder zu Doppelungen: Informationsportale wie die Deutsche Digitale Bibliothek, das Archivportal-D und das Deutsche Textarchiv werden im Kapitel „Bibliothek,

\footnotetext{
${ }^{27}$ Jannidis, Fotis/Kohle, Hubertus/Rehbein, Malte (Hrsg.): Digital Humanities. Eine Einführung, Metzler, Stuttgart 2017.

${ }^{28}$ Siehe die ausführliche Rezension von Stäcker, Thomas: Rezension zu „Digital Humanities. Eine Einführung", in: o-bib. Das offene Bibliotheksjournal (2017), H. 3, S. 142-148, URL: <https://www.o-bib.de/ article/view/2017H3S142-148/5979> [Zugriff 18.07.2020].

${ }^{29}$ Klinke, Harald: Aufbau des Computers und Vernetzung, in: Jannidis/Kohle/Rehbein (Hrsg.): Digital Humanities (wie Anm. 27), S. 35-58.

${ }^{30}$ Reichert, Ramón: Theorien digitaler Medien, in: Jannidis/Kohle/Rehbein (Hrsg.): Digital Humanities (wie Anm. 27), S. 19-35.

${ }^{31}$ Jannidis, Fotis: Grundlagen der Datenmodellierung, in: Jannidis/Kohle/Rehbein (Hrsg.): Digital Humanities (wie Anm. 27), S. 99-108.

${ }^{32}$ Volkmann, Armin: Geographische Informationssysteme, in: Jannidis/Kohle/Rehbein (Hrsg.): Digital Humanities (wie Anm. 27), S. 299-314.

${ }^{33}$ Website des Buches beim Verlag Metzler, URL: <http://www.metzlerverlag.de/9783476026224> [Zugriff: 15.08 .2020$]$.
} 
Archiv, Museum“ von Heike Neuroth ${ }^{34}$ genauso vorgestellt wie im anschließenden Kapitel „Aufbau von Datensammlungen“ von Christof Schöch ${ }^{35}$. Auch die vier Arten von Metadaten (deskriptiv, strukturell, administrativ und technisch) finden sich mehrfach im Band und mit unterschiedlicher Tiefe erläutert. Auffallend ist, dass trotz der großen Themenvielfalt eine theoretische Auseinandersetzung mit den epistemologischen Änderungen in den Geisteswissenschaften durch die Digitalisierung ebenso fehlt wie ein Beitrag zu den institutionellen und curricularen Entwicklungen der Digital Humanities in Deutschland, wobei die erste Lücke stärker schmerzt. ${ }^{36}$ Einige Überlegungen dazu finden sich gleichwohl im Artikel „Digital Humanities als Wissenschaft" von Manfred Thaller. ${ }^{37}$ Der emeritierte Professor und Doyen der deutschsprachigen digitalen Geschichtswissenschaft geht der Frage nach, ob die DH als Werkzeug oder als Methode zu begreifen sind. Zugespitzt lautet diese Frage für ihn, ob mit neuen Technologien dieselben Ergebnisse nur schneller und effizienter als in traditioneller Weise erzielt werden, oder ob neue und andere Ergebnisse erreicht werden können, die ohne Einsatz der IT nicht möglich wären (S. 13). Aus der Sicht von Thaller werden die DH für die Hermeneutik methodisch signifikant, ,,weil der Umgang mit digital gespeicherter Information oft leichter nachvollziehbar und für andere dokumentierbar ist" (S. 15). Somit ist die quantitative oder statistische Argumentation ein Beweislieferant. Für Thaller können quantitative Verfahren daher Forschungsgegenstände der Geisteswissenschaften ,intensiver und gründlicher“ (S. 18) untersuchen. Sie können aber zugleich auch wissenschaftstheoretische Fragen eröffnen, was allerdings nicht erläutert wird. Weitere Erkenntnisse könnte die im März 2020 erfolgte Gründung einer Arbeitsgruppe „Digital Humanities Theorie" im Verband der deutschsprachigen Digital Humanities liefern, die Theorie und Theoriebildung in den digitalen Geisteswissenschaften aus unterschiedlichen Disziplinen beleuchtet. ${ }^{38}$ Aber auch die geplante Neuauflage des Lehrbuchs könnte diese Leerstelle füllen.

\section{Für den Überblick: zwei Einführungen zur digitalen Geschichte}

Wer sich weniger mit digitaler Praxis und Informatik beschäftigen möchte, sondern sich stärker für ein Gesamtbild, Beispiele und für die digitalen Änderungen

\footnotetext{
${ }^{34}$ Neuroth, Heike: Bibliothek, Archiv, Museum, in: Jannidis/Kohle/Rehbein (Hrsg.): Digital Humanities (wie Anm. 27), S. 213-222.

35 Schöch, Christof: Aufbau von Datensammlungen, in: Jannidis/Kohle/Rehbein (Hrsg.): Digital Humanities (wie Anm. 27), S. 223-233.

${ }^{36}$ Zur institutionellen und curricularen Entwicklung der DH und digitalen Geschichte in Deutschland siehe Hohls, Rüdiger: Digital Humanities und digitale Geschichtswissenschaften, in: Busse, Laura u.a. (Hrsg.): Clio-Guide. Ein Handbuch zu digitalen Ressourcen für die Geschichtswissenschaften (Historisches Forum, Bd. 23), Berlin ${ }^{2} 2018$, URL: <https://guides.clio-online.de/guides/arbeitsformen-undtechniken/digital-humanities/2018?language=de $>$ [Zugriff: 31.07.2020]; Sahle: Digital Humanities (wie Anm. 6).

37 Thaller, Manfred: Digital Humanities als Wissenschaft, in: Jannidis/Kohle/Rehbein (Hrsg.): Digital Humanities (wie Anm. 27), S. 11-18.
}

38 AG Digital Humanities Theorie, URL: <https://dhtheorien.hypotheses.org/> [Zugriff: 18.07.2020]. 
allgemein interessiert, kann zur Einführung in die digitale Geschichte von Guido Koller, Historiker und Archivar im Schweizerischen Bundesarchiv, greifen. ${ }^{39}$ Der Untertitel seines 2016 publizierten und knapp 160 Seiten umfassenden Bandes ist vielversprechend: „Historische Welten neu vermessen“. Gemeint ist damit eine hybride Vorstellung von digitaler Geschichte, bei der die Vergangenheit in Form von historischen Quellen mit computergestützten Methoden vermessen und anschließend analog interpretiert wird. Somit bleibt dem Menschen die ,tiefe Interpretation von Texten und der Umgang mit Widersprüchlichkeiten in Quellen“ (S. 10) vorbehalten.

Im ersten Teil der Einführung geht es um den „Stand der Dinge“. Hier umreißt Koller auf knapp 70 Seiten die wesentlichen Änderungen, die die Geschichtswissenschaft durch die Digitalisierung erfahren hat. Sein Ansatz ist breit und er sieht die Produktion, Vermittlung und Vernetzung von historischem Wissen gleichermaßen durch den digitalen Wandel betroffen, der ,, auf die Voraussetzungen für das Schreiben von Geschichte zurück[wirkt]“ (S. 7). Einer der Schwerpunkte von Koller als Archivar ist die veränderte Rolle der Archive im digitalen Zeitalter vom gatekeeper zum data broker (S. 25), sowie die Schwierigkeit, die Authentizität und Integrität digitaler Quellen zu überprüfen. Insbesondere für die neueste Zeitgeschichte ändert sich die Quellenbasis durch genuin digitale Quellen fundamental. Zeitgeschichtsforschung findet daher häufig sehr zeitnah zu den Ereignissen statt, so Koller (S. 23), stehen doch zahlreiche Quellen sofort zur Verfügung. Zugleich stellt er die Frage, welche neuen Erkenntnisse sich durch die Analyse von Archivmaterialien nach Ablauf ihrer 30-jährigen Schutzfrist bieten werden. Wird man beispielsweise 2045 mehr über die Verhandlungen zum Schuldenabbau und den Reformen in Griechenland sagen können als 2015, oder sind Positionen und Argumente vor allem durch die sozialen und klassischen Medien bereits umfassend bekannt (S. 28)? Weitere Kapitel des ersten Teils gelten digitalen Infrastrukturen und Fallbeispielen aus der digitalen Geschichte, die das Erkenntnispotenzial computergestützter Methoden deutlich machen. Für Koller ist die deutschsprachige Geschichte in der Anwendung digitaler Methoden im Vergleich zur angloamerikanischen Geschichte sowie zu den Digital Humanities allgemein ein Nachzügler (S. $64 \mathrm{f}$.).

Zumeist sind Projekte, Methoden und Konzepte auf einfachem Niveau erklärt, manchmal sind die Texte jedoch nicht voraussetzungslos, etwa wenn Begriffe wie ,Post-GreSQL-Datenbank“ und „DBpedia 8“ [sic] fallen (S. 53), die zwar kursiv gesetzt, aber nicht weiter erklärt sind. So verständlich und didaktisch der Text an manchen Stellen ist - etwa bei den Überlegungen zur Analyse (S. 32) -, so vage bleibt er an anderen Stellen. Über die Publikationsplattform „OpenEdition“ heißt es beispielsweise, die Plattform ,ist um eine Kerngruppe herum organisiert, welche die Abläufe in der Community strukturiert" (S. 77).

Im zweiten Teil der Einführung geht es in sechs Kapiteln um die Perspektiven der digitalen Geschichte. Diese sind nicht einzelnen Methoden gewidmet, sondern betreffen wie bereits in Teil eins überwiegend die veränderte Arbeitsweise der historischen Zunft. Dazu gehören unter anderem die Arbeit bei „offener Werkstatt“ durch die Nutzung von Wissenschaftsblogs, die Pro-Amateur-Revolution, virtuelle Räume und Zeiten, die Hybridität von Medien und Methoden sowie ein Kapitel über die

39 Koller, Guido: Geschichte digital. Historische Welten neu vermessen, Kohlhammer, Stuttgart 2016. 
Philosophie der digital history. Wie Graham, Milligan und Weingart betont Koller, dass computergestützte und analoge Methoden sich nicht ausschließen, vielmehr gegenseitig ergänzen und gemeinsam eine „,doppelte Perspektive“ (S. 81) schaffen. Für Koller geht die digitale Geschichte damit über frühe Ansätze der hypertextuellen Darstellung von Geschichte hinaus, wie sie Wolfgang Schmale 2010 vertrat. $^{40}$ Stattdessen sei sie eine „Disziplin, welche widersprüchliche Entwicklungen in temporären (virtuellen) Räumen und an spezifischen Orten umfassend zu beschreiben, zu analysieren und zu verstehen versucht“ (S. 86). Mit nur etwas über 20 Seiten bleibt dieser Perspektiventeil sehr kurz. Ein Weiterdenken der möglichen Erkenntnispotenziale computergestützter Methoden anhand konkreter Anwendungsbeispiele oder Fragestellungen sowie eine Vertiefung der Gedanken zum Einfluss von Algorithmen auf Forschungsergebnisse und der Vorstellung von Tools als ,kognitive Schnittstellen“ (S. 67, 84) wären wünschenswert gewesen.

Der dritte Teil des Bandes ist mit „Serviceteil“ überschrieben und enthält auf knapp 40 Seiten eine Zusammenstellung von Kurzporträts zu einer Auswahl wichtiger Infrastrukturen, Projekten, Plattformen, Anwendungen, Standards, Zeitschriften und Blogs. Die Einteilung ist zwar nicht immer einleuchtend - etwa das Zusammenfassen von „Tools, Maschinenlesbaren Sprachen und Standards“ -, doch die Auswahl und die Kurztexte sind nützlich und verständlich erklärt, etwa die Begrifflichkeiten rund um das Thema digitale Inhaltsanalyse. Im Serviceteil werden zudem die für die Geschichtswissenschaft wichtigen digitalen Editionen aufgeführt, die man in den ersten beiden Teilen vermisst.

Am Ende der Lektüre bleibt trotz eines umfassenden Ansatzes und vieler Anregungen ein gemischter Eindruck, bedingt vor allem durch manche Vermischung von Ebenen, durch Wiederholungen und bisweilen ungenaue Zusammenhänge. Das zwar schöne Bild der historischen Welten, die digital zu vermessen und analog zu interpretieren sind, kommt in fast identischer Formulierung außer in der Einleitung zugleich auf den Seiten 90, 92 und 97 vor, die Kritik an Wolfgang Schmale zweimal (S. 39, 84). Das hätte einem Lektorat genauso auffallen müssen wie die durchgehende Bezeichnung ,Tweeds“ statt Tweets für Kurznachrichten bei Twitter und die durch ihre Häufigkeit störende Kursivsetzung von mittlerweile gebräuchlichen Wörtern wie Blogs und Code neben Eigennamen wie Google, Wikipedia, Twitter und Ähnlichem.

Wer Französisch liest, dem sei das Buch von Philippe Rygiel empfohlen, Professor für Zeitgeschichte an der École normale supérieure in Lyon und Spezialist für französische Migrationsgeschichte. Unter dem Titel „L'historien à l'age numérique" hat er 2017 eine Sammlung von 13 Aufsätzen herausgebracht, die er zwischen Mitte der 1990er Jahre und 2014 publiziert hat, ergänzt durch eine rund 30-seitige Einleitung. ${ }^{41}$ Einzelne Aufsätze sind leicht überarbeitet und sprachlich vereinfacht worden, jedoch ohne sie (oder ihre abgelaufene URL) zu aktualisieren. Vorangestellt ist ihnen jeweils ein kurzer Vorspann zu ihrem damaligen Entstehungskontext. Es ist erstaunlich, wie wenig den Aufsätzen ihr Alter anzumerken ist. Das deutet

\footnotetext{
40 Vgl. Schmale, Wolfgang: Digitale Geschichtswissenschaft, Böhlau, Wien u. a. 2010.

41 Rygiel, Philippe: Historien à l'âge numérique, Presses de l'Enssib, Lyon 2017, URL: <https://books. openedition.org/pressesenssib/6303> [Zugriff; 18.07.2020].
} 
zum einen darauf hin, dass es Rygiel weniger um die Praxis als um die Theorie der digitalen Geschichte geht. Zum anderen ist es ein Indiz dafür, dass viele Überlegungen zur theoretischen Einrahmung der digitalen Geschichte sowie Forderungen zum Umgang damit bereits seit 20 Jahren oder länger auf fast identische Weise angestellt werden, wobei die meisten Fragen weiterhin offen bleiben und es trotz wiederholten Debatten nicht zu produktiven Anpassungen im Fach gekommen ist.

Rygiel legt mit seinem Buch keine klassische Einführung in die digitale Geschichte vor. Vielmehr beschäftigt er sich mit den Änderungen, die der Beruf der Historikerin und des Historikers durch die digitale Transformation erfahren hat. Im Hinblick auf Qualität und Reichweite der Reflexionen ist sein Buch am ehesten mit „Digital Past“ vergleichbar, der Habilitationsschrift des leider viel zu früh verstorbenen Historikers Peter Haber. ${ }^{42}$ Ähnlich wie Haber geht es Rygiel darum, wie sich alltägliche Praktiken des Suchens und Verarbeitens von Informationen durch die Digitalisierung verändert haben, wie sich dabei die Beziehung der Forschenden zu den Quellen (ob digitalisiert oder genuin digital) gewandelt hat, wie sich das Schreiben von Geschichte im Internet verändert und welche Kenntnisse der Informatik und Statistik notwendig sind, um weiter mit der notwendigen und gewohnten Gründlichkeit historisch forschen, lehren und publizieren zu können. Rygiels feine Analysen zielen darauf, die enge Verflechtung der alltäglichen Praktiken der Historiker_innen mit der digitalen Welt zu zeigen. Das Digitale ist demnach nicht losgelöst von der Sphäre der Geschichtswissenschaft. Es ist nichts, gegen das man sein kann und umgekehrt auch nichts, in dem man aufgehen könnte. Vielmehr ist es die Textur selbst, innerhalb derer gegenwärtig Wissensgewinnung stattfindet (S. 18). Die sich schon früh abzeichnende zögerliche Haltung großer Teile der Zunft erscheint Rygiel damit unverständlich und unproduktiv, die Idee einer digitalen Geschichte von Spezialist_innen, die neben einer traditionellen und unveränderten Clio existiert, wenig überzeugend. Bei Rygiel steht damit nicht die computergestützte Geschichte im Mittelpunkt, sondern Geschichtswissenschaft im digitalen Zeitalter. Und so leuchtet er die epistemologischen Änderungen der digital gewordenen Rahmenbedingungen aus, die gegenwärtig für jede Form der Geschichte grundlegend sind.

Der Band ist in zwei Teile unterteilt. Die sechs Aufsätze im ersten Teil „Archéologie d'une pratique" (Archäologie einer Praxis) widmen sich vor allem der Planung und dem Aufbau von Webseiten, seien es Quellenportale, Instituts- oder Archivwebseiten, E-Journals, Diskussionsforen, Mailinglisten. Es ist die Sicht der späten 1990er Jahre, die hier deutlich wird, als manche Historiker_innen anfingen, eigene Webangebote aufzubauen, wobei sie mit ihren wissenschaftlichen Start-ups ganz neue Kenntnisse beherrschen mussten. Es war eine Zeit des Bastelns und Ausprobierens, noch weit entfernt von den später institutionalisierten großen Playern wie HumaNum und OpenEdition, die mittlerweile digitalisierte Quellen, Daten, Forschungsliteratur, Tools und Angebote zur Wissenschaftskommunikation bereitstellen. Die Beiträge in diesem Teil sind vielfach deskriptiv, enthalten aber zugleich ein Nachdenken über das digitalisierte Objekt als Repräsentation des ursprünglichen Archivdokuments (S. 60) oder Überlegungen zur Authentifizierung von genuin digitalen Quellen (S. 67). Als ein Akteur der ersten Internetstunde situiert Rygiel die

${ }^{42}$ Haber, Peter: Digital Past. Geschichtswissenschaft im digitalen Zeitalter, Oldenbourg, München 2011. 
Veränderungen damit in ihrer mittleren Dauer und zeigt, dass die Informatisierung der Welt weder plötzlich einsetzte noch stets gleichmäßig oder rasant verlief noch zukünftig verlaufen wird.

Besonders stark sind die Aufsätze im zweiten Teil des Bandes, der mit „Réflexivités réticulaires“ (Netzartige Reflexivitäten) überschrieben ist. Der digital turn ist für Rygiel vor allem ein reflexiver turn (,tournant réflexif“), womit er das Nachdenken über die digitalen Wissenspraktiken versteht. Wiederholt beschäftigt er sich beispielsweise mit Webarchiven und genuin digitalen Quellen, denen aus seiner Sicht - wenn sich nichts ändert - später die Historiker_innen fehlen werden, weil sie sich gegenwärtig nicht in die Debatten einmischen. ${ }^{43}$ Die Überlegungen Rygiels basieren zumeist auf konkreten Beispielen, was sie äußerst plastisch machen. So untersuchte er 2009 am Beispiel der Ausweisung von Einwanderer_innen in Frankeich der letzten 20 Jahre, welche Informationen dazu im Netz zu finden waren, die man nicht zugleich in der Presse oder in Archiven finden konnte (S. 131). Schnell werden in seinem Selbstversuch die Schwierigkeiten beim Finden, Zusammenstellen und Bewerten des Korpus deutlich: die hohe Anzahl an Ergebnissen, die Diversität der Formate und Inhalte, aber auch Zugangsbeschränkungen, da Teile des kulturellen Erbes in den Händen privater Firmen liegen, außerdem die Schwierigkeiten bei der Bewertung und Kritik der Authentizität und Integrität der Quellen, ihre instabile URL mit der Folge, dass bestimmte Quellen schon kurze Zeit später nicht mehr auffindbar waren et cetera. Zukünftige Historiker_innen, so Rygiel, haben kaum mehr eine Wahl und müssen ein Prozedere dafür entwickeln, die Masse an Dokumenten sinnvoll auswerten zu können, ohne die Anforderungen der Disziplin an Genauigkeit und Gründlichkeit zu vernachlässigen (S. 146).

Rygiel ist - das dürfte deutlich geworden sein - kein Digital-Apologet, sondern begleitet die Entwicklungen kritisch. Er schreibt genauso über die Schwierigkeiten der Digitalisierung, die etwa einen konstanten Bedarf bei Historiker_innen hervorruft, die eigenen Praktiken an sich verändernde und kaum beherrschte Wissensumgebungen anzupassen (S. 27). Ebenso unterstreicht er die Wichtigkeit, Auswahl, Zusammenstellung und Interpretation von Quellen nicht dem Kontext von Werkzeugen zu überlassen, um weiterhin die eigenen Forschungsfragen stellen und die eigenen Konzepte anwenden zu können (S. 35). Ein weiteres Problem ist aus seiner Sicht die schnelle Veränderung in der digitalen Welt, die „nouveauté permanente“ (permanente Neuheit) (S. 160), durch die historisch Forschende zumeist alleine vor der Aufgabe stehen, sich neue Werkzeuge beizubringen, neue Software zu lernen und damit mühsam Kompetenzen anzueignen, die schon bald wieder obsolet werden (S. 162).

In fast allen Beiträgen kommt Rygiel daher auf die Notwendigkeit zurück, die universitäre Ausbildung von Historiker_innen den veränderten digitalen Rahmenbedingungen anzupassen und gemeinsam darüber nachzudenken, welchen Stellenwert

\footnotetext{
${ }^{43}$ Vgl. auf deutscher Seite jüngst dazu Bischoff, Frank M./Patel, Kiran Klaus: Was auf dem Spiel steht. Über den Preis des Schweigens zwischen Geschichtswissenschaft und Archiven im digitalen Zeitalter, in: Zeithistorische Forschungen 17 (2020) H. 1, S. 145-156, URL: <https://zeithistorische-forschungen.de/ 1-2020/5822> [Zugriff: 18.07.2020]. Auch hier erlaube ich mir, auf einen meiner Beiträge hinzuweisen: König, Mareike: Geschichtsforschung und Archive im digitalen Zeitalter. Chancen, Risiken und Nebenwirkungen, in: Der Archivar (2020), H. 4 (im Druck).
} 
IT-, Mathematik- und Statistikkenntnisse einnehmen sollten. Während Faktenvermittlung an Wert verliere, gewinne in einer Welt der Fake News die seit jeher traditionelle Kompetenz der Historiker_innen wieder an Bedeutung, verlässliche Informationen in Dokumenten zu finden, zu bewerten und zu organisieren (S. 176). Wichtig ist dabei aus der Sicht von Rygiel, die Lehre nicht auf technische Kenntnisse oder Tools auszurichten, die schnell wieder veralten, sich verändern oder ersetzt werden. Stattdessen sollte Raum für Methodik und Abstraktion bleiben. Rygiel fordert zugleich eine Anpassung der Präsentationsformen von Geschichte. Diese solle sich nicht hinter geschlossenen Mauern und in Büchern und Artikeln abspielen, sondern eine Präsenz in der öffentlichen Sphäre suchen (S. 178), in animierten Karten, Datenbanken, Simulationen, Wikis, Ausstellungen, Filmen, Spielen und anderen Verbreitungsmöglichkeiten, um das Interesse der Öffentlichkeit an Geschichte zu bedienen und damit die gesellschaftliche Relevanz des Fachs zu betonen (S. 21).

\section{Zur Übung: ein Praxisband Digital Humanities}

Rein auf die praktischen Seiten der Digital Humanities ausgerichtet ist der französische Band „Expérimenter les humanités numériques“, herausgegeben von den beiden Historikern Frédéric Clavert und Olivier Legendre, dem Bibliothekar Étienne Cavalié und - endlich eine Frau auf dem Buchtitel! - der Historikerin Dana Martin. ${ }^{44}$ Anhand konkreter Fallstudien möchte das Herausgeberteam aufzeigen, wie digitale Projekte entwickelt und wie dabei mit Herausforderungen umgegangen wird. Die verschränkten Präsentationen von digitalen Werkzeugen und Projekten dienen als Hilfestellungen und sollen Forschende als „kaleidoskopische Momentaufnahmen von tatsächlich erlebten Erfahrungen“ (instantané kaléidoscopique d'éxpériences réellement vécues) (S. 10) anregen, ihre Vorbehalte gegenüber digitalen Tools abzulegen. Insgesamt zwölf über einen Call eingeworbene Beiträge umfasst die $\mathrm{Pu}-$ blikation, wovon sieben im Band abgedruckt, die anderen wie auch die Bibliografie ausschließlich online zu finden sind. So sehr man sich über die Open-Access-Publikation freut, so unpraktisch ist der Medienbruch durch die Trennung der zitierten Literatur von den gedruckten Beiträgen: Wer die im gedruckten Band grundsätzlich abgekürzten Publikationstitel auflösen möchte, muss stets parallel die OnlineFassung aufrufen.

Die Publikation ist in drei Teile untergliedert und umfasst unter der Überschrift „Persönliche Werkzeuge“ (Les outils personnels) Beispiele für die Video-Annotation, Wissenschaftsblogs, soziale Medien, eine Datenbank aus den Theaterwissenschaften sowie eine Anleitung für die Literaturverwaltungssoftware Zotero. Teil zwei ist den „Kollektiven Werkzeugen“ (L'outillage collectif) gewidmet mit einem Beitrag zur Datenbank-Software Omeka, die unter anderem für Online-Ausstellungen eingesetzt wird, zu Wikis als Recherchetool und zu digitalen Editionen. In Teil drei schließlich geht es um die „Organisation von Forschungsprojekten“ (Gestion

\footnotetext{
44 Cavalié, Étienne u.a. (Hrsg.): Expérimenter les humanités numériques. Des outils individuels aux projets collectifs, PU Montréal, Montreal 2017, URL: <http://www.parcoursnumeriques-pum.ca/experimenter leshumanitesnumeriques $>$ [Zugriff: 18.07.2020].
} 
de projet) mit Beiträgen zum Forschungsdatenmanagement, zu GIS, zu gemeinsam geführten Datenbanken und zum Einsatz eines Gemeinschaftsblogs für die Projektorganisation. Die Aufteilung ist nicht ganz stringent, der ein oder andere Artikel hätte auch in einen anderen Teil verschoben werden können, was jedoch nicht der zentrale Kritikpunkt an dieser Publikation ist. Limitiert ist vor allem der Fokus der digitalen Methoden, die ganz überwiegend aus den Bereichen der Verbreitung und der Forschungsorganisation kommen, dagegen kaum Verfahrensweisen zur computergestützten Interpretation und Analyse von Quellen enthalten. Die Definition von Digital Humanities ist entsprechend dem Ansatz des Buches auf die Anwendung digitaler Tools in den Geistes- und Sozialwissenschaften beschränkt (S. 11), wonach die Reflexion dieser Praktiken und der Digitalisierung in ihren epistemologischen Auswirkungen nicht dazugehören, obwohl sie in den einzelnen Beiträgen durchaus vorkommen. Rhetorisch ist die Einleitung auf die Überredung zögernder Forschender ausgerichtet, was der Band allerdings mit manchen anderen Werken gemeinsam hat. Nutzen und Fruchtbarkeit digitaler Werkzeuge werden lediglich behauptet, nicht aber belegt. So heißt es etwa auf Seite $12 \mathrm{zu}$ den Digital Humanities: „die Ergebnisse sind beeindruckend“ (les résultats sont remarquables), ohne dies näher auszuführen oder ein Beispiel dafür zu nennen. Ob solche Aussagen Zögernde oder Kritiker_innen überzeugen, digitale Tools auszuprobieren, sei dahingestellt. Positiv zu vermerken ist, dass Anwendungstools gezeigt werden, für die es zumeist keine tieferen IT-Kenntnisse braucht, was - wie angestrebt - die Nutzungsschwelle bei Forschenden senken dürfte, sich an dieser Form der digitalisierten Geschichte zu beteiligen.

Der Gesamteindruck des Bandes ist - wie häufig bei Sammelbänden - gemischt; nicht alle Beiträge sind gleich gut dokumentiert oder erscheinen unmittelbar nützlich. Es zeigt sich zudem die Schwierigkeit, Projekt- und Toolvorstellung ansprechend zu verknüpfen, zumal wenn mehrere Tools hintereinander vorgestellt werden. Gelungen und empfehlenswert sind der einleitende Artikel zur Literaturverwaltungssoftware Zotero von Chloée Fabre ${ }^{45}$ sowie die sehr präzise Schritt-für-Schritt-Anleitung für ein Forschungsdatenmanagement von Aurore Cartier, Magalie Moysan und Nathalie Reymonet ${ }^{46}$. Der Software Omeka sind gleich zwei Beiträge von sehr unterschiedlicher Qualität gewidmet: ${ }^{47}$ Eher unentschieden in der Einschätzung des Nutzens von Omeka sind Cécile Boulaire und Romeo Carabelli. Anschaulich können die Autoren jedoch zeigen, dass Daten eben nicht einfach „gegeben“ sind, wie man im Französischen meinen könnte, wo Daten ,données“ heißen. Vielmehr gilt: „Les données ne sont pas données“ (S. 91). Stattdessen werden Daten fabriziert, indem sie aus Quellen extrahiert und geordnet werden. Sie sind damit das Ergebnis eines

\footnotetext{
45 Fabre, Chloée: Zotero. La gestion de références bibliographiques et de corpus documentaires, in: Cavalié u.a. (Hrsg.): Expérimenter (wie Anm. 44), nur online: URL: <http://parcoursnumeriques-pum.ca/ zotero-la-gestion-de-references-bibliographiques-et-de> [Zugriff: 18.07.2020].

46 Cartier, Aurore/Moysan, Magalie/Reymonet, Nathalie: Gestion des données, partage et conservation pérenne avec la Data Management-Plan, in: Cavalié u. a. (Hrsg.): Expérimenter (wie Anm. 44), nur online: URL: <http://parcoursnumeriques-pum.ca/gestion-des-donnees-partage-et-conservation-perenne-avecle> [Zugriff: 18.07.2020].

47 Boulaire, Cécile/Carabelli, Romeo: Du digital naive au bricoleur numérique. Les images et le logiciel Omeka, in: Cavalié u. a. (Hrsg.): Expérimenter (wie Anm. 44), S. 81-103.
} 
Prozesses, dem bestimmte Entscheidungen zugrunde liegen, und sind folglich nicht neutral. ${ }^{48}$ Präziser ist der Aufsatz von Fatiha Idmhand, Claire Riffard und Richard Walter, die Omeka für die Edition von modernen Handschriften verwendet haben. ${ }^{49}$ Der Beitrag zu den Sozialen Medien von Emmanuel Mourlon-Druol ${ }^{50}$ enthält zwar sinnvolle praktische Hinweise, wie etwa den, dass bereits auf einem Repository mit Zugriffszähler hinterlegte Artikel nicht zusätzlich bei Nicht-Repositorien wie dem ohnehin umstrittenen Academia.edu ${ }^{51}$ oder ResearchGate eingestellt werden sollten, um die Zugriffsstatistik nicht zu verfälschen. Doch bleibt der Text bis auf einen kurzen Abschnitt zu Twitter weitgehend leblos, da er lediglich Dienste aufzählt, ohne diese, ihre Anwendung oder gar den konkreten Nutzen für Forschende näher zu erläutern.

\section{Definitionsfragen, Aushandlungen und Kontroversen}

Wichtige Anregungen finden Historikerinnen und Historiker in den beiden umfangreichen Sammelbänden „Debates in the Digital Humanities“, herausgegeben von Matthew Gold und Lauren Klein ${ }^{52}$ (hybride, sowohl gedruckt als auch im Open Access online zugänglich), sowie „A New Companion to Digital Humanities“, herausgegeben von Susan Schreibman, Ray Siemens und John Unsworth ${ }^{53}$, aus denen hier bereits zitiert wurde. Beides sind publizistische Erfolge: die „Debates“ erscheinen nach 2012, 2016 und 2019 voraussichtlich 2021 zum vierten Mal, der „Companion“ von 2016 ist die zweite Ausgabe nach der von 2004, der das Feld seinen Namen Digital Humanities zu verdanken hat. ${ }^{54}$ An der Fortschreibung der Inhaltsverzeichnisse lässt sich die Entwicklung der Digital Humanities nachvollziehen. War in der Ausgabe der „Debates“ von 2012 noch ein eigener Abschnitt dem Thema „Definition“ gewidmet, so liegt der Schwerpunkt der Ausgabe von 2016 unter dem Untertitel „Expanded field“ auf der thematischen und methodischen Offenheit der $\mathrm{DH}$, die sich engen Definitionen entziehe. Zugleich wird darin kritisch Stellung gegenüber der vielfach zitierten Vorstellung der Digital Humanities als „großes Zelt““ (big tent) genommen, das in der Praxis eben doch nicht unterschiedslos allen Perso-

\footnotetext{
48 Siehe dazu auch Fickers: Update (wie Anm. 19).

49 Idmhand, Fatiha/Riffard, Claire/Walter, Richard: L'édition électronique de manuscrits modernes, in: Cavalié u. a. (Hrsg.): Expérimenter (wie Anm. 44), S. 105-123.

50 Mourlon-Druol, Emmanuel: L'usage des réseaux sociaux pour chercheurs, in: Cavalié u.a. (Hrsg.): Expérimenter (wie Anm. 44), S. 37-53.

51 Demantowsky, Marko: Bei Academia.edu etc. bleiben? Wissenschaftliche Textrepositorien haben auch ihre Geschichte, in: Digital Humanities am DHIP, 8. März 2019, URL: <https://dhdhi.hypotheses.org/ 5861> [Zugriff: 31.07.2020].

52 Gold, Matthew K./Klein, Lauren F. (Hrsg.): Debates in the Digital Humanities 2016, Minnesota UP, Minneapolis, MN/London 22016, URL: <https://dhdebates.gc.cuny.edu/projects/debates-in-the-digitalhumanities-2016> [Zugriff: 18.07.2020].

53 Schreibman, Susan/Siemens, Ray/Unsworth, John (Hrsg.): A New Companion to Digital Humanities (Blackwell Companions to Literature and Culture), Wiley-Blackwell, West Sussex u. a. ${ }^{2} 2016$.

54 Der Band von 2004 ist mittlerweile online frei zugänglich: dies. (Hrsg.): A Companion to Digital Humanities, URL: <http://www.digitalhumanities.org//companion/> [Zugriff: 18.07.2020].
} 
nen gleichermaßen offensteht. So gibt es eigene Abschnitte zur Kritik an den Digital Humanities sowie zur Zukunft der DH. Darin geht es unter anderem um das Manifest der \#transformDH-Bewegung, die dafür eintritt, dass Forschung und Lehre in den Digital Humanities Rassismus, Genderfragen und Behinderungen thematisieren sowie auf soziale Gerechtigkeit, freien Zugang und Inklusion zielen sollten. ${ }^{55}$

Die anders als im deutsch- und französischsprachigen Raum grundlegend politische Haltung der DH in den USA wird in der neuesten Ausgabe der „Debates“ von 2019 weiter verdeutlicht: „A DH that matters“ lautet der Untertitel und so plädieren die Herausgebenden dafür, dass die Digital Humanities zu einem stärker technischen und historisch informierten Widerstand gegen gegenwärtige politische Tendenzen nach der Wahl von Donald Trump zum US-Präsidenten beitragen sollten. Als zentral wird die gesellschaftliche Bedeutung der DH angesehen, die in der Forschung und in ihren Projekten deutlich werden müsse, etwa durch das Aufdecken rassistischer Verzerrungen durch Algorithmen in Suchmaschinen. ${ }^{56}$

Während in den 2000er und frühen 2010er Jahren also das Neue und Vielversprechende, die Potenziale und Möglichkeiten, das Transformative des digital turn betont wurden und man sich gegen Anfeindungen von außen wappnete, ob real oder imaginiert, richtet sich die Diskussion in den Digital Humanities gegenwärtig stärker nach innen und auf dortige Spannungen. Dazu gehört etwa die Kontroverse, ob man Digital Humanist sein kann, auch wenn man selbst nichts Digitales erschafft, sondern sich ausschließlich der Theorie widmet - als ,hack vs. yack“ wird diese Kontroverse oft abgekürzt. ${ }^{57}$ Definitionsfragen werden eher auf der Ebene der Methoden und Praktiken verhandelt, etwa im „New Companion“ von Lorna Hughes, Panos Constantopoulos und Costis Dallas in ihrem sehr informativen Artikel zu Taxonomien und Ontologien computergestützter Methoden in den Geisteswissenschaften. ${ }^{58}$ Die Ausdifferenzierung und Vertiefung mancher Themen und Methoden machen es notwendig, die Subtilitäten und fachgebundenen Charakteristiken des eigenen geisteswissenschaftlichen Fachs den anderen Fächern zu erläutern, um eine unerwünschte Aufspaltung der Digital Humanities abzuwenden. Die Kritik an den Digital Humanities hat gleichwohl über die Jahre nicht nachgelassen. ${ }^{59}$

Nach wie vor ist auch das Verhältnis zwischen digitaler Geschichte und Digital Humanities nicht immer spannungsfrei: Im Vergleich zu Sprach- und Literaturwis-

\footnotetext{
55 Bailey, Moya u.a.: Reflections on a Movement: \#TransformDH, Growing Up, in: Gold/Klein (Hrsg.): Debates 2016 (wie Anm. 52), S. 71-79. \#TranformDH ist auch im „New Companion“ Gegenstand der Betrachtungen, z. B. Warwick, Claire: Building Theories or Theories of Building. A Tension at the Heart of Digital Humanities, in: Schreibman/Siemens/Unsworth (Hrsg.): New Companion (wie Anm. 53), S. 538-552.

56 Gold, Matthew K./Klein, Lauren F.: Introduction, in: dies. (Hrsg.): Debates in the Digital Humanities 2019, Minnesota UP, Minneapolis, MN/London ${ }^{3} 2019$, URL: <https://dhdebates.gc.cuny.edu/projects/ debates-in-the-digital-humanities-2019> [Zugriff: 18.07.2020].

57 Z. B. Nowviskie, Bethany: On the Origin of "Hack" and "Yack", in: Gold/Klein (Hrsg.): Debates 2016 (wie Anm. 52), S. 66-70; Warwick: Building Theories (wie Anm. 55), hier S. 541.

58 Hughes, Lorna/Constantopoulos, Panos/Dallas, Costis: Digital Methods in the Humanities. Understanding and Describing Their Use Across the Disciplines, in: Schreibman/Siemens/Unsworth (Hrsg.): New Companion (wie Anm. 53), S. 150-170.

59 Gold, Matthew K./Klein, Lauren F.: Introduction, in: dies. (Hrsg.): Debates 2016 (wie Anm. 52), S. xiii.
} 
senschaften - so heißt es oft, aber unbelegt - sei die digitale Geschichte in den DH weniger gut repräsentiert. Außerdem laufe die Geschichtswissenschaft Gefahr, ihre Eigenheiten in der doppelten Transdisziplinarität der Digital Humanities (zur Informatik sowie zwischen den geisteswissenschaftlichen Fächern) aufzulösen. Stephen Robertson geht in einem Beitrag in den „Debates“ von 2016 der Frage nach, wie sich die digitale Geschichte von den Digital Humanities abgrenzen lässt. ${ }^{60}$ Ähnlich wie Philippe Rygiel betont er die Wichtigkeit eines genuin historischen Zugangs bei der Verwendung digitaler Methoden, unterscheiden sich die geisteswissenschaftlichen Fächer doch durch ihre eigenen Quellen, Fragestellungen und Herangehensweisen (S. 289). Aus seiner Sicht wirken sowohl die Interdisziplinarität der DH als auch das Digitale auf große Teile der Zunft abschreckend (S. 290) und so plädiert er dafür, das vermeintlich ,große Zelt“ der DH eher als ein Haus mit vielen verschiedenen Zimmern zu sehen (S. 291). Einen Unterschied macht der Professor an der George Mason University und frühere Direktor des Roy Rosenzweig Center for History and New Media in der unterschiedlichen Verwendung des Web aus: Historiker_innen nutzen es anders als etwa Computerlinguist_innen, und zwar eher, um mit ihren Themen und Forschungen in die Klassenzimmer zu gelangen und die breite Öffentlichkeit zu erreichen (S. 291 ff.). Bei den digitalen Methoden liegt der bisherige Schwerpunkt der historisch Forschenden seiner Analyse nach auf GIS und spatial mapping (S. 293-296), weniger auf Textanalyse, weil - im Unterschied zur gedruckten Prosa des 18. und 19. Jahrhunderts, die der digitalen Literaturwissenschaft als Rohstoff für ihre Forschungen dient - weniger digitalisierte und im Volltext erschlossene handschriftliche Archivquellen zur Verfügung stehen (S. 296). Hinzu kommen, so Robertson, beschränkte Zugänge auf proprietäre Angebote sowie fehlende Schnittstellen und Downloadmöglichkeiten, um überhaupt die Möglichkeit zu haben, Digitalisate anders als durch bloßes Lesen auszuwerten (S. 297).

Der Mangel an digital auswertbaren Quellen als Grund für die Zurückhaltung der historischen Forschung beim Einsatz digitaler Methoden ist ein Argument, das auch Guido Koller vertritt (S. 65). Nun hat jedoch der Pool an Online-Archivquellen durch zahlreiche große Digitalisierungsprojekte sowie durch genuin digitale Quellen in den letzten Jahren stark zugenommen. Sicherlich eignen sich nicht alle Quellen gleichermaßen für eine digitale Analyse, doch wird ihre Verfügbarkeit zukünftig weiter exponentiell ansteigen. Aus meiner Sicht hat die Zurückhaltung in der historischen Zunft bei der Nutzung digitaler Methoden insbesondere für die Textanalyse weitere Gründe, zum Beispiel das weitverbreitete Ablehnen quantitativer Methoden; mangelnde IT-Kenntnisse; unklare Aussichten, welche Ergebnisse die zumeist anspruchsvollen digitalen Projekte bringen; der große Aufwand allein dafür, ein digital untersuchbares Datenset zu erstellen; sowie vor allem fehlendes digitales Denken, um historische Fragestellungen zu entwickeln, die man an ein großes Textkorpus richten könnte. Hier bleibt unter anderem auf den Aufbau einer nationalen Forschungsdateninfrastruktur (NFDI) zu hoffen wie auf die zunehmende Vermittlung entsprechender digitaler Kompetenzen in der universitären Lehre und bei den Lehrenden selbst. Doch trotz der Zunahme an digitalisierten und aufbereiteten Quellen und der zu erwartenden Flut an genuin digitalen Quellen, sei es im Web oder in

60 Robertson: Differences (wie Anm. 24). 
Archiven, wird in naher Zukunft der Anteil derjenigen Forschenden, die computerbasierte Methoden in der Geschichtswissenschaft einsetzen, vermutlich sehr klein bleiben. Aus der Sicht von Ted Underwood werden dies in zehn Jahren 3 Prozent, optimistisch vielleicht 5 Prozent der Geisteswissenschaftler_innen sein. Als Ursache dafür sieht er nicht eine bewusste digital-feindliche Einstellung der Forschenden, sondern den momentanen Stand der universitären Ausbildung, die diese Bereiche nicht abdeckt, sodass sie auch nicht zur Anwendung kommen können. ${ }^{61}$

Eine weitere Kontroverse der letzten Jahre ist die kritische Verhandlung, was die digitale Geschichte mit ihren großen und teuren Projekten eigentlich geleistet habe. Ihr wird vorgeworfen, bisher nicht aus dem Stadium des ,ewigen Versprechens "62 herausgetreten zu sein, was Cameron Blevins in seinem Beitrag in den „Debates“ von 2016 diskutiert. „Potenzial“, „Möglichkeiten“ und „,neu“ seien die am häufigsten verwendeten Worte in Artikeln über digitale Geschichte, die damit sprachlich in einer ,ewigen Zukunftsform“ (perpetual future tense) feststecke (S. 309). Als Gründe dafür führt Blevins neben dem noch jungen Alter der digitalen Geschichte auch ihren Fokus auf sich wandelnde Technologien an, sodass immer etwas ,neu' sei. Zugleich könne hinter dieser Wortwahl auch eine Verkaufsstrategie im Hinblick auf Forschungsförderung stehen. Der wichtigste Grund für diesen Eindruck ist aus seiner Sicht jedoch, dass die digitale Geschichte sich bisher zu wenig mit neuen Ergebnissen und Argumenten an Forschungsdiskursen beteiligt hat (ebd.). Das Kerngeschäft der Geschichtswissenschaft sei es, zu argumentieren, während die digitale Geschichte ihre Energie in die Online-Stellung von Quellen und den Aufbau von Plattformen und Tools gesteckt habe. Zwar beinhalteten auch diese eine Interpretation, aber nicht im klassischen Sinne ein Argument, wie es in der Geschichtsforschung insbesondere im Hinblick auf Karrierewege erwartet und bewertet werde. Die Gründe dafür sieht er zum einen im ,langen Schatten“ der quantitativen Geschichte in den 1960er und 1970er Jahren, der sich hartnäckig als Bild einer positivistischen Geschichtsschreibung hält (S. 310). ${ }^{63}$ Daraus sei bei digital arbeitenden Historiker_innen eine bis heute spürbare Angst vor einer argumentativen Überdehnung auf der Basis numerischer Beweise entstanden (S. 311) ${ }^{64}$ Darüber hinaus habe sich in der anschließenden Phase der public history alles um die Präsentation von Quellen und Forschungsergebnissen gedreht, sodass die Beteiligung am Forschungsdiskurs weiterhin keine Priorität hatte (S. 312). Wie schon Tom Scheinfeldt in den ,Debates“ 201265, vertritt

\footnotetext{
61 Underwood, Ted: Digital Humanities as a Semi-Normal Thing, in: Gold/Klein (Hrsg.): Debates 2019 (wie Anm. 56), URL: <https://dhdebates.gc.cuny.edu/read/untitled-f2acf72c-a469-49d8-be3567f9ac1e3a60/section/ac5fc1c4-abcb-4a04-8a4b-fa5fe763220e\#ch10> [Zugriff: 18.07.2020].

62 Blevins, Cameron: Digital History's Perpetual Future Tense, in: Gold/Klein (Hrsg.): Debates 2016 (wie Anm. 52) S. 308-324, URL: <http://dhdebates.gc.cuny.edu/debates/text/77> [Zugriff: 18.07.2020].

63 So auch Hohls: Digital Humanitites (wie Anm. 36).

64 Siehe dazu die Untersuchung über den Einsatz quantitativer Methoden in ausgewählten historischen Fachzeitschriften von Buchner, Michael u.a.: Zur Konjunktur des Zählens - oder wie man Quantifizierung quantifiziert. Eine empirische Analyse der Anwendung quantitativer Methoden in der deutschen Geschichtswissenschaft, in: Historische Zeitschrift 310 (2020), H. 3, S. 580-621.

65 Scheinfeldt, Tom: Where's the Beef? Does Digital Humanities Have to Answer Questions?, in: Gold, Matthew K. (Hrsg.): Debates in the Digital Humanities, Minnesota UP, Minneapolis, MN/London 2012, URL: <http://dhdebates.gc.cuny.edu/debates/text/18> [Zugriff: 18.07.2020].
} 
Blevins folglich die These, dass digitale Historiker_innen sich sehr viel stärker im Bereich der argumentativen Forschung engagieren müssen, um mehr Anerkennung im Fach zu erhalten. Dabei sollen die Bereiche der Bereitstellung und Erschließung von Quellen nicht aufgegeben werden. Die Diskussion darüber wurde 2017 in einem white paper des Roy Rosenzweig Center for History and New Media vertieft. ${ }^{66}$

Ähnliche Überlegungen finden sich bei William G. Thomas III, Historiker an der University of Nebraska-Lincoln, in seinem Aufsatz im „New Companion“.67 Auch er konstatiert, dass die DH zwischen 1993 und 2013 relativ wenig interpretative oder argumentative Wissenschaft produziert habe, wie sie in den Geisteswissenschaften üblicherweise diskutiert, bewertet und rezensiert wird (S. 525). Eines der Hauptprobleme macht er in der anhaltenden Kultur der Monografie aus, wie sie vor allem die Geschichtswissenschaft pflegt, die aber als Endergebnis für digitale Forschung nicht angemessen ist. Dadurch werden digitale Arbeiten nicht indexiert oder rezensiert und damit auch nicht von den traditionell arbeitenden Teilen der Zunft wahrgenommen, mit entsprechenden Risiken für jüngere Forschende, die computergestützt arbeiten oder digitale Projekte erschaffen möchten. Dahinter steckt die nicht mehr ganz neue, aber weitgehend ungelöste Frage, wie digitale Anteile an - zumal kollaborativen Forschungsarbeiten angemessen bewertet werden können. Notwendig sind aus der Sicht von Thomas neue digitale Narrative für die computergestützte Forschung, die nicht in gedruckter Form erscheinen kann. Gemeint sind damit keine bloßen E-Books, die das analoge Buch in ein digitales Umfeld transferieren, sondern neue Formate: nichtlineare, multimodale Erzählungen mit expliziter Hyptertext-Struktur, die mehrere Einstiegsmöglichkeiten und multiple Lesewege erlauben (S. 532).

Demgegenüber geht der amerikanische Historiker Lincoln A. Mullen in seinem Beitrag in den „Debates“ von 2019 wieder einen Schritt zurück mit seiner These, dass für die Präsentation von digitalen Forschungsergebnissen Prosa nach wie vor die beste Variante bleibe, trotz aller Experimente mit neuen Formaten und den Fortschritten bei Visualisierungen. ${ }^{68}$ Als Formel schlägt er eine ,,verflochtene Erzählung“ (braided narrative) vor. Mullen knüpft an die Beobachtung an, dass digitale Historiker_innen viel über ihre Methoden schreiben, aber wenig argumentative Wissenschaft fabrizieren. Andersherum schreiben traditionell arbeitende Historiker_innen wenig oder gar nicht über ihre Methode und verstecken diese sogar eher. Folglich gäbe es einen Mangel an Interpretation in der digitalen Geschichte und einen Mangel an expliziter methodologischer Diskussion in der traditionellen Geschichte. Aus der Sicht von Mullen ist es daher zentral, die historische Interpretation und Argumentation in den Mittelpunkt der Geschichtsschreibung zu stellen und gleichzeitig der Erläuterung der Methodik, die zu diesen Ergebnissen geführt hat, gebührend Gewicht beizumessen. Es gelte, Interpretationen und Methodendiskussion in einem gemeinsamen Text

\footnotetext{
66 Arguing with Digital History Working Group: Digital History and Argument White Paper. Roy Rosenzweig Center for History and New Media 2017, URL: <https://rrchnm.org/argument-white-paper/> [Zugriff: 18.07.2020].

67 Thomas III, William G.: The Promise of the Digital Humanities and the Contested Nature of Digital Scholarship, in: Schreibman/Siemens/Unsworth (Hrsg.): New Companion (wie Anm. 53), S. 524-537.

68 Mullen, Lincoln A.: A Braided Narrative for Digital History, in: Gold/Klein (Hrsg.): Debates 2019 (wie Anm. 56), URL: <https://dhdebates.gc.cuny.edu/read/untitled-f2acf72c-a469-49d8-be35-67f9ac1e3a60/ section/e5496dc6-dcc1-42e7-8609-9377d05812c5\#ch31> [Zugriff: 18.07.2020].
} 
zu verweben sowie Code und Daten in entsprechenden Repositories frei zugänglich und nachnutzbar zu machen.

Man darf gespannt sein, wie das Fach auf die hier angesprochenen Problematiken und Kontroversen in naher Zukunft reagieren wird. Dies gilt insbesondere für die enormen Herausforderungen bei der Ausbildung der zukünftigen Historiker_innen, aber auch für die epistemologischen Fragen dieses reflexiven turns, in dem sich die Geschichtswissenschaft seit einigen Jahrzehnten befindet. Die hier vorgestellten Publikationen zeigen, welche Veränderungen sich seit den Anfängen der cliometrics bereits eingestellt haben und sie zeigen genauso, wo die Diskussionen bisher stecken geblieben sind. Ihr Verdienst ist es, die Herausforderungen, vor dem das Fach als Ganzes steht, kontinuierlich in Erinnerung zu rufen. Zu hoffen bleibt, dass sie viele Leserinnen und Leser finden.

\section{Besprochene Literatur}

- Cavalié, Étienne u.a. (Hrsg.): Expérimenter les humanités numériques. Des outils individuels aux projets collectifs, 166 S., PU Montréal, Montreal 2017, URL: <http://www.parcoursnumeriques-pum.ca/experimenterleshumanitesnumeriques> [Zugriff: 18.07.2020].

- Gold, Matthew K./Klein, Lauren F. (Hrsg.): Debates in the Digital Humanities 2016, 600 S., Minnesota UP, Minneapolis, MN/London 22016, URL: <https:// dhdebates.gc.cuny.edu/projects/debates-in-the-digital-humanities-2016> [Zugriff: 18.07.2020].

- Gold, Matthew K./Klein, Lauren F. (Hrsg.): Debates in the Digital Humanities 2019, 472 S., Minnesota UP, Minneapolis, MN/London ${ }^{32019, ~ U R L: ~<h t t p s: / / ~}$ dhdebates.gc.cuny.edu/projects/debates-in-the-digital-humanities-2019> [Zugriff: 31.07.2020].

- Graham, Shawn/Milligan, Ian/Weingart, Scott: Exploring Big Historical Data. The Historian's Macroscope, 282 S., ICP, London 2015, URL Preprint Version: $<$ http://www.themacroscope.org/> [Zugriff: 18.07.2020].

- Jannidis, Fotis/Kohle, Hubertus/Rehbein, Malte (Hrsg.): Digital Humanities. Eine Einführung, 370 S., Metzler, Stuttgart 2017.

- Koller, Guido: Geschichte digital. Historische Welten neu vermessen, 157 S., Kohlhammer, Stuttgart 2016.

- Rygiel, Philippe: Historien à l'âge numérique, 208 S., Presses de l'Enssib, Lyon 2017, URL: <https://books.openedition.org/pressesenssib/6303> [Zugriff: 18.07.2020].

- Schreibman, Susan/Siemens, Ray/Unsworth, John (Hrsg.): A New Companion to Digital Humanities (Blackwell Companions to Literature and Culture), 586 S., Wiley-Blackwell, West Sussex u. a. ${ }^{2} 2016$.

Funding Open Access funding enabled and organized by Projekt DEAL.

Open Access Dieser Artikel wird unter der Creative Commons Namensnennung 4.0 International Lizenz veröffentlicht, welche die Nutzung, Vervielfältigung, Bearbeitung, Verbreitung und Wiedergabe in 
jeglichem Medium und Format erlaubt, sofern Sie den/die ursprünglichen Autor(en) und die Quelle ordnungsgemäß nennen, einen Link zur Creative Commons Lizenz beifügen und angeben, ob Änderungen vorgenommen wurden.

Die in diesem Artikel enthaltenen Bilder und sonstiges Drittmaterial unterliegen ebenfalls der genannten Creative Commons Lizenz, sofern sich aus der Abbildungslegende nichts anderes ergibt. Sofern das betreffende Material nicht unter der genannten Creative Commons Lizenz steht und die betreffende Handlung nicht nach gesetzlichen Vorschriften erlaubt ist, ist für die oben aufgeführten Weiterverwendungen des Materials die Einwilligung des jeweiligen Rechteinhabers einzuholen.

Weitere Details zur Lizenz entnehmen Sie bitte der Lizenzinformation auf http://creativecommons.org/ licenses/by/4.0/deed.de.

Dr. Mareike König hat Geschichte, Germanistik und Politikwissenschaft in Hamburg und Paris studiert und wurde in Rostock promoviert. Sie hat außerdem ein Studium der Bibliotheks- und Informationswissenschaften in Berlin absolviert. Ihre Forschungsschwerpunkte liegen auf der deutsch-französischen Geschichte des 19. Jahrhunderts sowie auf der digitalen Geschichte. Sie ist stellvertretende Direktorin am Deutschen Historischen Institut in Paris, wo sie zugleich die Abteilung Digital Humanities und die Bibliothek leitet. 